\title{
BMJ Open Quality A multifaceted intervention to improve the quality of care for patients undergoing total joint arthroplasty
}

\author{
Lyle Sorensen, ${ }^{1}$ Lori Idemoto, ${ }^{2}$ Janet Streifel, ${ }^{1}$ Barbara Williams, ${ }^{2}$ \\ Robert Mecklenburg, ${ }^{2}$ Craig Blackmore ${ }^{2}$
}

To cite: Sorensen L, Idemoto L, Streifel J, et al. A multifaceted intervention to improve the quality of care for patients undergoing total joint arthroplasty. BMJ Open Quality 2019;8:e000664. doi:10.1136/ bmjoq-2019-000664

Received 20 February 2019 Revised 17 April 2019 Accepted 19 June 2019

\section{Check for updates}

(C) Author(s) (or their employer(s)) 2019. Re-use permitted under CC BY-NC. No commercial re-use. See rights and permissions. Published by BMJ.

${ }^{1}$ Department of Orthopedic Surgery, Seattle, Washington, USA

${ }^{2}$ Center for Health Care Improvement Science, Virginia Mason Medical Center, Seattle, Washington, USA

Correspondence to Dr Craig Blackmore; craig.blackmore@ virginiamason.org

\section{ABSTRACT}

Knee and hip arthroplasties vary in cost, quality and outcomes. We developed a Lean quality improvement intervention for knee and hip arthroplasty patients encompassing the recognition, readiness, restoration and recovery phases of care.

The intervention included standardised, evidence-based pathways, shared decision making, patient and family member engagement, and transdisciplinary rounding, implemented successively through a series of rapid process improvement workshops. We evaluated the intervention through run charts and time series analysis for 2005-2014. Outcomes included length of stay (LOS), 30-day readmission, discharge disposition, postsurgical complications and patient satisfaction. Included were 4253 total joint arthroplasty procedures, 1659 hip and 2594 knee. LOS decreased from 3.2 to 2.4 days postintervention for both hip and knee patients $(p<0.001)$. The 30-day hospital readmission rate for hip patients decreased from $3.1 \%(18 / 576)$ to $1.1 \%(5 / 446$, $p=0.032$ ) with knee patients unchanged. Discharge to home (vs rehabilitation facility or skilled nursing facility) increased from $72 \%(415 / 576)$ to $91 \%(405 / 446)$ $(\mathrm{p}<0.001)$ for hip patients, and from $70 \%(599 / 860)$ to $87 \%(578 / 663)$ for knee patients $(p<0.001)$.

Our standardised multifaceted Lean quality improvement programme was associated with reduced LOS, decreased readmission rates and improved discharge disposition in total knee and hip arthroplasty patients.

\section{PROBLEM}

We identified significant variability in the care of hip and knee arthroplasty patients that presented improvement opportunities. Further, we anticipated increased growth of these procedures over the next several years. Accordingly, in 2010, we initiated a multifaceted Lean quality improvement intervention focused on improving the medical care and patient experience for individuals undergoing total hip arthroplasty (THA) and total knee arthroplasty (TKA). The objective of this report was to detail the effect of this quality improvement intervention on patient care.

The intervention took place at a tertiary referral 336-bed hospital in the Pacific Northwest with 16500 inpatient admissions and
7600 inpatient operating room procedures in 2014. Annual procedure volumes averaged 520 for knee arthroplasty and 330 for hip arthroplasty. Physician assistants assisted surgeons during arthroplasty procedures and follow-up inpatient care.

Our institution deploys the Lean production principles of waste elimination from the Toyota Production System applied to healthcare. ${ }^{1}$ A key attribute of this system is integration of quality improvement into the daily work, achieved through invested leadership that helps to create and maintain a culture including a shared vision and goals. ${ }^{2}$ Quality improvements are achieved through engaging all stakeholders, including frontline workers and patients, in process improvement events to improve the design of care.

\section{BACKGROUND}

Knee and hip arthroplasties are common procedures that are increasing annually due to the ageing 'baby boomer' population worldwide, increased access to care, particularly under the US Affordable Care Act, and improved diagnosis and treatment. ${ }^{3}{ }^{4}$ Estimates from the US National Inpatient Sample and census bureau population statistics predict growth in total hip revisions and total knee revisions by $137 \%$ and $601 \%$, respectively, between 2005 and $2030 .{ }^{35}$ Currently, there is wide variation in cost, outcomes and criteria to perform these procedures. ${ }^{6-8}$

Increasing emphasis on quality and changing payment models is compelling a shift from volume to value-based healthcare, and organisations will need to provide the best patient experience and outcomes while reducing cost and waste. ${ }^{4}$ Bundled payments and warranties exemplify this shift and encourage hospitals and providers to work together toward reliable and predictable service. ${ }^{910}$ TKA and THA surgeries are typically elective procedures designed to relieve pain and improve physical function, 
and patients often have choices about where to have the surgery. ${ }^{11} 12$ These attributes, combined with projected growth, make arthroplasty ideal for value-based care models that promote use of evidence-based criteria, shared decision making and improvement in the patient's experience. $^{13}$

\section{MEASUREMENT}

To determine intervention effectiveness, we performed a retrospective time series study for 2005-2014. Eligible patients had Common Procedure Terminology codes for TKA (27447) and for THA (27130). Excluded were subjects who underwent bilateral procedures, those admitted through urgent care or the emergency department (including fractures) and those with metastatic cancer affecting the bone.

The primary outcome was length of stay (LOS). Secondary outcomes were 30-day readmission, patient discharge disposition, postsurgical complications (postoperative hypotension (any systole $\leq 90$ ), postoperative anaemia and code-like medical emergency team (MET) calls) and patient satisfaction. Data were extracted from the electronic medical record for all outcomes, except for MET call data, which were obtained from MET activation logs, and patient satisfaction data, which were obtained from third-party administered surveys of all patients discharged from the hospital. These surveys were part of standard hospital patient satisfaction monitoring, and third-party survey administrators were not involved in the data analysis, reporting of the results or review of this paper. The surveys were mailed or emailed to all patients 2-4 days postdischarge. We used three questions as the survey outcomes: whether the patients would recommend the hospital to others, whether the patients felt their pain was adequately controlled and whether the patient felt ready for discharge. Responses were on a 5-oint scale, with 1 being 'very poor' and 5 being 'very good'. The survey response rate was $28.5 \%(739 / 2594)$ for TKA patients and $29.5 \%(489 / 1659)$ for THA patients.

For 2005-2009, data were available only for LOS and readmissions. More detailed data on surgical outcomes were available starting in 2010. Preoperative risk factors analysed included comorbidities defined by International Classification of Diseases, Ninth Revision (ICD9), codes for hypertension (401.9), diabetes (250.00) and high cholesterol (272.4). Obesity was identified by a body mass index (BMI) of $\geq 30$, and metabolic syndrome was defined by a BMI of $\geq 30$ with ICD9 diagnoses of diabetes and high cholesterol.

The time period 2005-2011 was the baseline, with 2012 and 2013 serving as the intervention period and 2014 serving as the follow-up (sustainability) period. Though the quality improvement work began in 2010, broad implementation across the hospital and providers did not occur until 2012-2013. The t-test was used to compare means, and the $\chi^{2}$ to compare proportions before and after the intervention. In addition, the data were analysed graphically using statistical process control charts. To adjust for any effect of patients with lower BMI postintervention, we performed a subanalysis limited to patients with a BMI of <30. We also performed stepwise linear regression to control for the effects of hypertension, diabetes, hypercholesterolaemia, BMI, age and gender on the primary outcome of LOS. Statistical analysis was performed using StataMP V.12.0. This study was a quality improvement project and was determined to be exempt from review by our institutional review board.

\section{DESIGN}

Our vision was to create a patient-centred, personalised, comfortable and transparent journey for the patient from the time that a decision to perform surgery was considered until 12 weeks after discharge. This vision was formulated in 2010 through a multistakeholder Rapid Process Improvement Workshop (RPIW), with participation from orthopaedic surgery, primary care, nursing, anaesthesia, physical therapy and hospital management. Experience-based design techniques provided information from patients and staff on where to focus process improvement efforts. ${ }^{1415}$ From this event, we developed a fourphase care model for TKA and THA patients consisting of recognition, readiness, restoration and recovery. A key attribute of this model was a flow process that focused on the progression of the patient and not the location within the health system.

Each RPIW had a standard structure, with predefined targets. The organisation of each event was a standard teaching curriculum for participants, root cause analysis, idea generation, solution development and finally, trial implementation. The second half of each event was focused on plan do study act (PDSA) cycles of the changes proposed. The 30 days postevent emphasised implementation of the changes, with formal report out to the entire organisation at 30 and 90 days. These report outs emphasised successes and challenges with implementation, as well as reporting on the individual event targets. In addition, there was monthly tracking of the primary outcome, LOS. This was displayed publically on a visibility wall throughout the entire project. The experience at each event served to inform planning of the subsequent events throughout the 3-year project time cycle.

\section{STRATEGY}

We initiated the arthroplasty quality improvement intervention in April 2010 and continued through full implementation in 2013 (figure 1).

Implementation of each component of the programme occurred through a series of RPIWs of 2 or 5 days focused on a specific operational area (figure 1). PDSA cycles were completed to support implementation of each improvement effort. Data on the primary outcomes were tracked in real time with team huddles on a weekly basis. We also had regularly report-out sessions to the institution as a whole 90 days after each RPIW. 

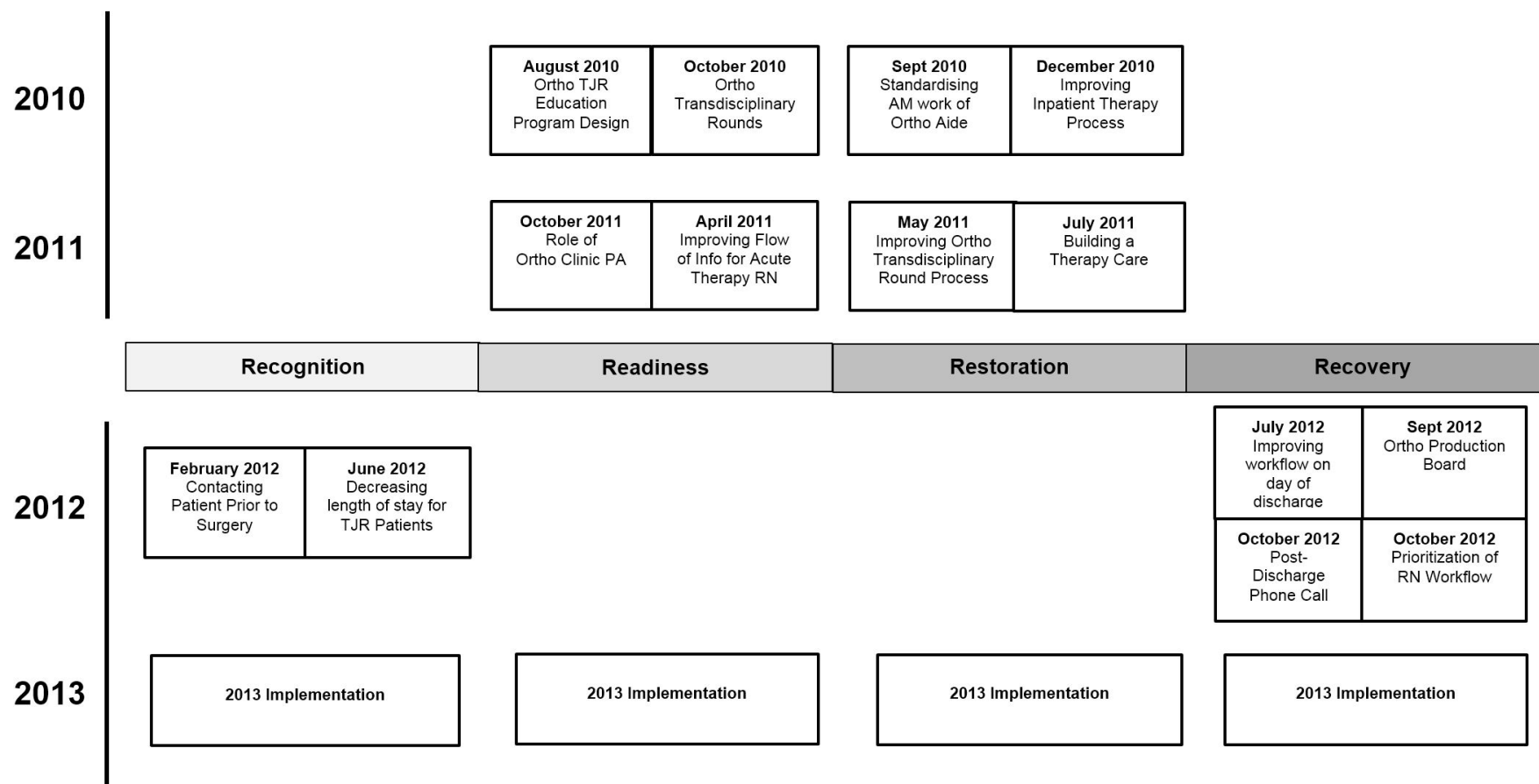

Figure 1 Timeline of quality improvement events in the lean quality improvement intervention. PA, physician assistant; RN, registered nurse; TJR, total joint replacement.

\section{Phase 1: recognition}

Recognition consisted of integration of a standardised, evidence-based approach across primary care and radiology to identify individuals who would potentially benefit from arthroplasty versus conservative care. Radiologists were directed to add the Kellgren-Lawrence Osteoarthritis Score to all relevant knee and hip radiograph reports to enable triage of subjects who should be referred to the arthroplasty surgeon directly. Patients with less disability and less severe osteoarthritis were managed primarily with a conservative care pathway with surgeon referral if conservative care failed or if high-risk conditions were present (repetitive falls, pain scale continually greater than 6 out of 10 , or continued joint effusions or haemarthrosis). The intent was to avoid referring patients to the surgeon who were not reliably surgical candidates without exhausting conservative measures first. Evidence-based criteria were also deployed to direct toward non-operative therapy patients who were at high surgical risk. ${ }^{16}$

\section{Phase 2: readiness}

Readiness spanned the time from the initial arthroplasty surgeon visit until the day of surgery and included the presurgical assessment, and patient engagement and preparation. We implemented shared decision making, a collaborative decision-making process between patient, health coach nurse and provider, using surgery risk algorithms to inform patients of the risks and benefits involved. ${ }^{11}$ Readiness also included a preoperative class to coach patients regarding expectations during hospitalisation and early recovery. Patients identified a care partner who committed through a signed contract to the support and the recovery of the patient after discharge. The preoperative evaluation was improved by combining patient engagement counselling and discussions with the care partner, lab draws, and preoperative physical therapy teaching and evaluation, into a single visit. Further, physical therapy consultation was added preoperatively to enable the initiation of rehabilitation education before the patient was limited by postoperative pain. We also implemented a preoperative checklist of all elements of patient care necessary to assess medical and emotional surgery readiness (cessation of smoking, management of high body mass index and control of diabetes). ${ }^{16}$

\section{Phase 3: restoration}

Restoration extended from surgical admission until discharge. Restoration phase work focused on improving the patient experience and decreasing LOS. Through root cause analysis, we identified that pain, hypotension and nausea contributed to longer stays and worse patient experience. Pain control was improved by implementing adductor canal blocks instead of femoral nerve blocks, with resultant preserved quadriceps function. ${ }^{17}$ In hip patients, we added local anaesthetic wound infiltration to decrease the need for systemic narcotics. Hypotension, in addition to contributing to longer LOS and increased fall risk, created a perceived 'negative' hospital experience. Before our improvement efforts, hypotensive episodes led to 'code-like' MET activations, contributing to patient anxiety, fear about their clinical condition and reluctance to be discharged. Accordingly, we created a nurse-initiated fluid bolus protocol to prevent significant hypotensive events. At the same 
time, we initiated the use of an intraoperative fibrinogen inhibitor (tranexamic acid) to minimise bleeding and transfusion requirements, potentially decreasing postoperative hypotension.

To address nausea, we added antiemetics to the preanaesthesia and postanaesthesia regimens, decreased narcotic usage and decreased blood loss from use of tranexamic acid. ${ }^{18}$ These factors were designed to improve functional status, enabling ambulation on the day of surgery and increasing physical therapy to two times per day. We also designed an innovative 'therapy car' to help patients simulate getting in and out of a real vehicle. The therapy car was created from plastic plumbing tubing and connectors and was designed to adjust to simulate different types of vehicles. In addition, we posted a 'milestones to recovery' mobility chart in patients' rooms to engage patients in achieving the functional status required for discharge.

In addition, we deployed transdisciplinary team morning rounds to guide care and to identify patients off the recovery pathway. Other components of standard care included evidence-based venous thromboembolism prophylaxis, antibiotic prophylaxis and surgical site infection prevention measures imbedded within the order sets. Finally, we tracked on the ward electronic census board, candidates for early discharge ( $<60$ hours) who were emotionally and functionally prepared for discharge.

\section{Phase 4: recovery}

The recovery phase spanned the time from home discharge until 12 weeks later. The preparation for rapid recovery was initiated during the readiness and restoration phases, with early physical therapy education, identification of potential early discharge patients and identification of a care partner described previously. Patients were encouraged to engage in self-directed home exercise for the first week after discharge and then to transition to outpatient physical therapy when the wound was stable and healing. Postdischarge care was supported with a standardised follow-up nurse phone call of 48-72 hours.

\section{RESULTS}

During the study period 2010-2014, 4253 total joint arthroplasty procedures, 1659 THAs (576 before, 637 during and 446 after) and 2594 TKAs (860 before, 1071 during and 663 after) were included (table 1A,B). An additional 2591 procedures were performed between 2005 and 2009. There were no significant differences in age before and after the intervention, but the proportions of patients with hypertension, diabetes, hypercholesterolaemia, high BMI and metabolic syndrome were significantly lower after the intervention (table 1A,B).

The average LOS decreased from 3.2 days in 2010 to 2.4 days in 2014 for both THA (figure 2A and table 2A) and TKA (figure 2B and table 2B) patients. For hip patients, the absolute change was 0.78 days (CI 0.68 to $0.95, \mathrm{p}<0.001)$ and for knee patients, the change was 0.78 days (CI 0.68 to $0.88, \mathrm{p}<0.001$ ). The proportion of patients with LOS of 4 or more days decreased from $19 \%(110 / 576)$ to $9 \%(42 / 446)$ in the hip patients $(\mathrm{p}<0.001)$ and from $19 \%(164 / 860)$ to $10 \%(63 / 663)$ in the knee patients $(\mathrm{p}<0.001)$. For hip patients with a BMI of $<30$, the LOS decreased from 3.2 to 2.4 days, a difference of 0.88 days (CI 0.64 to $1.11 \mathrm{p}<0.001$ ), while for knee patients with a BMI of $<30$, the LOS decreased from 3.2 to 2.4 days, a difference of 0.79 days (CI 0.63 to $0.94, \mathrm{p}<0.001)$. Overall, the mean LOS for patients who were readmitted within 30 days was 3.4 days $(n=51$, $\mathrm{SD}=1.3$, CI 3.0 to 3.8), while the mean LOS for patients who were not readmitted was 2.8 days $(\mathrm{n}=2494, \mathrm{SD}=1.2$, CI 2.8 to $2.9, \mathrm{p}<0.001$ ). For both hip and knee patients, both subanalysis limited to patients with a BMI of $<30$ and regression adjusting for any effects of hypertension, diabetes, hypercholesterolaemia, BMI, age and gender confirmed similar decreases in LOS, suggesting that a change in the sample population from 2010 to 2014 did not account for the observed improvements.

Discharge disposition to home or home health service improved for both hip and knee patients. For hip patients, disposition to home (vs rehabilitation facility or skilled nursing facility) increased from 72\% (415/576) in 2010 to $91 \%(405 / 446)$ in $2014(\mathrm{p}<0.001)$. For knee patients, disposition to home increased from $70 \%$ (599/860) to $87 \%(578 / 663)$ during that same period $(\mathrm{p}<0.001)$. There were no deaths in the hospital at any time during the study time frame.

The 30-day hospital readmission rate for hip patients decreased from $3.1 \%(18 / 576)$ to $1.1 \%(5 / 446, \mathrm{p}=0.032)$, with knee patients maintaining a $2 \%$ rate before $(16 / 860)$ and after the intervention $(12 / 663, \mathrm{p}=0.94)$. For all patients in the baseline and postintervention groups combined, the readmission rate for patients who were discharged to home was $1.4 \%$ (29/1997), while the readmission rate for patients who were discharged to a skilled nursing facility was $5.3 \%(22 / 416, \mathrm{p}<0.001)$.

The number of hip patients with postoperative hypotension decreased from $16 \%(90 / 576)$ to $4 \%(16 / 446$, $\mathrm{p}<0.001)$ and that in knee patients decreased from $9 \%$ $(75 / 860)$ to $2 \%(14 / 663, \mathrm{p}<0.001)$. There was also a significant decrease in MET calls for hip patients $(5 \%$, $27 / 576$ to $2 \%, 9 / 446, \mathrm{p}=0.022$ ), while there was no change in MET calls for knee patients at 3\% (22/860 before and 18/663 after the intervention, $\mathrm{p}=0.85$ ). Posthaemorrhagic blood loss was significantly improved $(\mathrm{p}<0.001)$ in both hip and knee patients.

For hip patients, there was a significant increase in 'strongly agree' with recommendation of the hospital from $79 \%(127 / 161)$ to $95 \%(103 / 109) \quad(p<0.001)$. For knee patients, the increase in 'strongly agree' with recommendation of the hospital was significant, from $83 \%(200 / 240)$ to $91 \%(148 / 163) \quad(p=0.032)$. Knee patients also reported a significant improvement in 'very good' pain control, from 65\% (155/240) at 
Table 1A Orthopaedics total hip replacement, descriptives for years 2010-2014

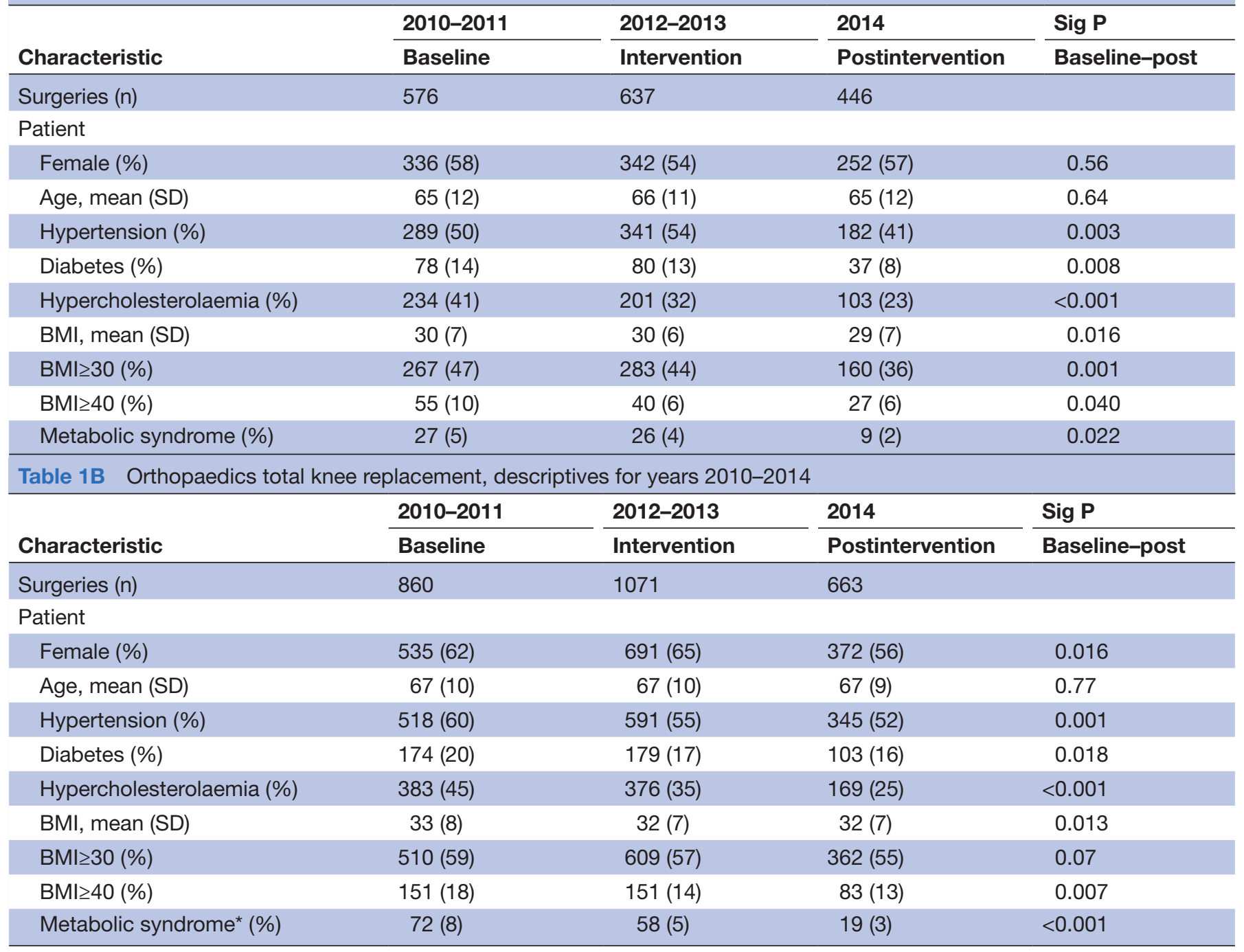

${ }^{*}$ Metabolic syndrome is defined by $\mathrm{BMI} \geq 30$ and diagnosis of hypertension, diabetes and high cholesterol.

BMI, body mass index; Sig, significant.

baseline to $74 \%(132 / 179)$ postintervention $(\mathrm{p}=0.046)$. The difference in hip patients was not significant, from $74 \%(114 / 155)$ to $79 \%(92 / 116) \quad(p=0.27)$. Patients' feelings of being ready for discharge were not significantly changed in either hip or knee patients, when comparing responses before and after the intervention.

\section{LESSONS AND LIMITATIONS}

In this work, we address the care of patients with arthritis spanning initial presentation in primary care through arthroplasty and recovery. Rather than structure the project around the silos of medical care delivery (eg, primary care, orthopaedics and physical therapy), we sought to consider the comprehensive care pathway from the patient's perspective. This was determined up front, with the broad vision accepted by all stakeholders and with buy-in from hospital management. Through the long course of the project, this shared vision allowed repeated efforts, even when success did not occur initially. It took iteration through 14 RPIWs on different phases of the project before the results were sufficient to support dissemination. Without the initial work aligning stakeholders, we do not believe we could have sustained the work long enough to realise success. The identification of a guiding team, with executive-level responsibility and a unifying vision, provided the essential foundation that allowed us to sustain the effort and was probably more critical to the success than any of the specific improvement events.

Our project does have limitations. The institutional Lean culture may not exist in all organisations. Also, the interventions extended over 2010-2013 and continued, making it difficult to assess the impact of each individual component. We were unable to collect information on implementation retrospectively. Our study occurred at a single teaching hospital, which may limit generalizability. In addition, the ability to use standardised, evidencebased criteria and processes may be challenging for other institutions that do not have an integrated health system. Finally, there is a potential for cost savings through 

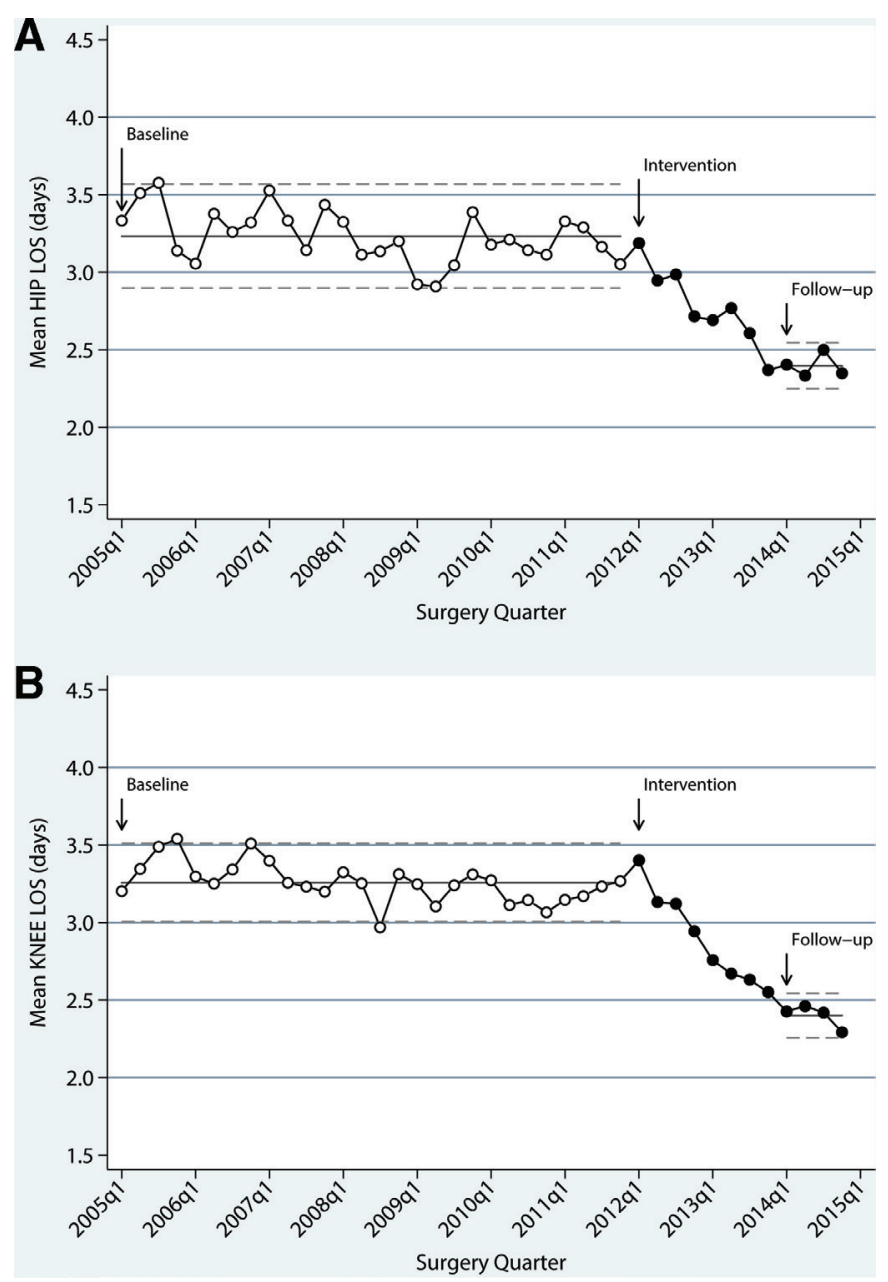

Figure 2 (A) Statistical process control chart for mean LOS for hip replacement by quarter. $n=2665$ hip replacements. See figure 1 for a more detailed timing of the interventions. (B) Statistical process control chart for mean LOS for knee replacement by quarter. $n=4179$ knee replacements. See figure 1 for a more detailed timing of the interventions. LOS, length of stay.

decreased LOS, decreased readmission and increase in discharge disposition directly to home. However, we were not able to assess all costs incurred to determine if cost savings were realised due to these improvements.

Finally, there are limitations that could confound the analysis. The use of appropriateness criteria for surgical intervention selected healthier patients for surgery and could contribute to improved outcomes. Pugely et al reported that comorbidities, most commonly hypertension, diabetes and obesity, were associated with increased LOS. ${ }^{19}$ Metabolic syndrome can also affect arthroplasty surgical complication rate and LOS. ${ }^{20}{ }^{21}$ However, neither analysis adjusting for these comorbidities nor limitation of the study to subjects with a BMI of $<30$ changed the results, indicating that selection criteria alone do not explain our improved outcomes. In addition, the number of patients with metabolic syndrome in our study was small $(<10 \%)$ and would not be expected to affect the findings. Our study does, however, support the use of appropriateness criteria in total joint arthroplasty to assure cost-effective and value-added use of healthcare resources. ${ }^{5}$

\section{CONCLUSION}

In this paper, we demonstrate the use of Lean to address the multicomponent care of patients with arthritis of the knee and hip, extending from initial non-operative treatment in primary care through surgical intervention, through recovery. Standardisation and improvement of care across the disease spectrum required collaboration between primary care, orthopaedic surgery, radiology, anaesthesia, nursing, pharmacy and physical therapy, all with agreed-upon standards of care in this population. We demonstrate that despite increasing $(11 \%-15 \%)$ numbers of total knee and hip arthroplasties, from 2010 to 2014, implementation of a multifaceted Lean quality improvement intervention was associated with sustained improvements in LOS, readmissions, discharge disposition and patient satisfaction. The key themes that contributed to this success were the use of standardised, evidence-based protocols, increased efficiency of processes and improvement in patient engagement. Our results also showed an inverse relationship between LOS and rate of readmission.

A comprehensive arthroplasty care pathway from the decision to have surgery until recovery is vital to improved outcomes. ${ }^{11} 2223$ Shared decision making during the presurgical visit increases patient knowledge and understanding to create more accurate expectations, increased engagement and greater satisfaction. ${ }^{11}$ Our surgical criteria included radiographs to determine the physical extent of cartilage loss, but also disability assessment, and determination of patient emotional readiness and comorbidities. Presurgical education and physical therapy were directed at decreasing anxiety and aided preparation for earlier rehabilitation. Visible surgical recovery goals helped patients and families see progress and feel empowered to engage in recovery. An important learning was that patients must be not only physically but also emotionally and practically ready for discharge. Implementation was aided through the use of Lean improvement events that focused on specific segments of the pathway and facilitated team building and monitoring for sustained improvement.

Our results are consistent with and build on previous reports. Compliance with evidence-based perioperative medications (antibiotic and venous thromboembolism prophylaxis) and standardised postoperative care have been shown to reduce LOS. ${ }^{1024}$ Other authors have shown success with clinical care pathways but have focused on the hospital only, rather than the complete care from primary care presentation through postsurgical recovery. ${ }^{19}$ 25-27 Prior work has shown that patients who were discharged earlier did not have an increased risk of readmission and that the proportion of patients discharged directly home increased with reduction of LOS. ${ }^{28}$ Further, patients who 
Table 2A Outcomes for orthopaedic total hip replacement, 2010-2014

\begin{tabular}{|c|c|c|c|c|}
\hline & 2010-2011 & 2012-2013 & 2014 & Sig $\mathbf{P}$ \\
\hline Characteristic & Baseline & Intervention & Postintervention & Baseline-post \\
\hline \multicolumn{5}{|l|}{ Surgical outcomes } \\
\hline LOS, mean (SD) & $3.2(1.2)$ & $2.8(1.0)$ & $2.4(1.5)$ & $<0.001$ \\
\hline LOS for $\mathrm{BMI}<30$ & $3.2(1.3)$ & $2.7(1.0)$ & $2.3(1.6)$ & $<0.001$ \\
\hline LOS for $\mathrm{BMI} \geq 30$ & $3.1(1.0)$ & $2.8(1.0)$ & $2.5(1.4)$ & $<0.001$ \\
\hline LOS $\geq 4$ days $(\%)$ & $110(19)$ & $86(14)$ & $42(9)$ & $<0.001$ \\
\hline 30-day readmission $(\%)^{*}$ & $18(3)$ & $13(2)$ & $5(1)$ & 0.032 \\
\hline Any systole $\leq 90(\%)$ & $90(16)$ & 37 (6) & $16(4)$ & $<0.001$ \\
\hline Posthaemorrhagic anaemia (\%)† & $471(82)$ & $519(81)$ & $254(57)$ & $<0.001$ \\
\hline Discharge status (\%) & & & & $<0.001$ \\
\hline Home/home health service & $415(72)$ & $500(78)$ & $405(91)$ & \\
\hline Skilled nursing & $134(23)$ & $119(19)$ & $39(9)$ & \\
\hline Other & $27(5)$ & $18(3)$ & $2(1)$ & \\
\hline MET call, any (\%) & $27(5)$ & $32(5)$ & $9(2)$ & 0.022 \\
\hline Operating room minutesł, mean (SD) & $98(39)$ & $93(36)$ & $84(22)$ & 0.031 \\
\hline \multicolumn{5}{|l|}{ Patient Satisfaction Survey (five-point scale) } \\
\hline \multicolumn{5}{|l|}{ Recommend hospital } \\
\hline Mean (SD) & $4.71(0.67)$ & $4.85(0.56)$ & $4.90(0.47)$ & 0.013 \\
\hline Very good (\%) & $127(79)$ & $183(90)$ & $103(95)$ & $<0.001$ \\
\hline \multicolumn{5}{|l|}{ Pain controlled } \\
\hline Mean (SD) & $4.65(0.69)$ & $4.73(0.64)$ & $4.75(0.57)$ & 0.21 \\
\hline Very good (\%) & $114(74)$ & $164(80)$ & $92(79)$ & 0.27 \\
\hline \multicolumn{5}{|l|}{ Ready for discharge } \\
\hline Mean (SD) & $4.63(0.64)$ & $4.75(0.54)$ & $4.66(0.62)$ & 0.78 \\
\hline Very good (\%) & $112(70)$ & $163(80)$ & $83(72)$ & 0.72 \\
\hline \multicolumn{5}{|c|}{ Table 2B Outcomes for orthopaedic total knee replacement, 2010-2014 } \\
\hline & 2010-2011 & 2012-2013 & 2014 & Sig $\mathbf{P}$ \\
\hline Characteristic & Baseline & Intervention & Postintervention & Baseline-post \\
\hline \multicolumn{5}{|l|}{ Surgical outcomes } \\
\hline LOS, mean (SD) & $3.2(0.9)$ & $2.9(1.0)$ & $2.4(1.1)$ & $<0.001$ \\
\hline LOS for $\mathrm{BMI}<30$ & $3.2(0.9)$ & $2.9(1.1)$ & $2.4(1.1)$ & $<0.001$ \\
\hline LOS for $\mathrm{BMI} \geq 30$ & $3.2(0.9)$ & $2.9(0.9)$ & $2.4(1.0)$ & $<0.001$ \\
\hline LOS $\geq 4$ days $(\%)$ & $164(19)$ & $147(14)$ & $63(10)$ & $<0.001$ \\
\hline 30 -day readmission $(\%)^{*}$ & $16(2)$ & $20(2)$ & $12(2)$ & 0.94 \\
\hline Any systole $\leq 90(\%)$ & $75(9)$ & $53(5)$ & $14(2)$ & $<0.001$ \\
\hline Posthaemorrhagic blood loss (\%)† & $707(82)$ & $852(80)$ & $351(53)$ & $<0.001$ \\
\hline Discharge status (\%) & & & & $<0.001$ \\
\hline Home/home health service & $599(70)$ & $775(72)$ & $578(87)$ & \\
\hline Skilled nursing & $171(20)$ & $219(20)$ & $72(11)$ & \\
\hline Other & $90(10)$ & $77(7)$ & $13(2)$ & \\
\hline MET call, any (\%) & $22(3)$ & $49(5)$ & $18(3)$ & 0.85 \\
\hline Operating room minutes $\ddagger$, mean (SD) & $94(31)$ & $96(41)$ & $89(29)$ & 0.29 \\
\hline \multicolumn{5}{|l|}{ Patient Satisfaction Survey (5-point scale) } \\
\hline \multicolumn{5}{|l|}{ Recommend hospital } \\
\hline Mean & $4.79(0.53)$ & $4.88(0.35)$ & $4.88(0.43)$ & 0.07 \\
\hline
\end{tabular}

Continued 
Table 2B Continued

\begin{tabular}{|c|c|c|c|c|}
\hline & 2010-2011 & 2012-2013 & 2014 & Sig $P$ \\
\hline Characteristic & Baseline & Intervention & Postintervention & Baseline-post \\
\hline Very good (\%) & $200(83)$ & $270(89)$ & $148(91)$ & 0.032 \\
\hline \multicolumn{5}{|l|}{ Pain controlled } \\
\hline Mean & $4.55(0.72)$ & $4.67(0.60)$ & $4.68(0.64)$ & 0.06 \\
\hline Very good (\%) & $155(65)$ & $219(72)$ & $132(74)$ & 0.046 \\
\hline Mean & $4.62(0.58)$ & $4.71(0.53)$ & $4.56(0.80)$ & 0.35 \\
\hline Very good (\%) & $158(66)$ & $231(75)$ & $124(70)$ & 0.44 \\
\hline
\end{tabular}

*30-day hospital readmission for any admissions to urgent care or emergency department only.

†Posthaemorrhagic blood loss defined by discharge International Classification of Diseases, Ninth Revision, diagnosis of 285.1.

$\ddagger$ Available only for a subset of patients with specific employers $(\mathrm{N}=277)$.

BMI, body mass index; LOS, length of stay; MET, medical emergency team; Sig, significant.

were discharged to skilled nursing facilities had a higher readmission risk. ${ }^{29}$

Using a standardised, multifaceted and multidisciplinary Lean quality improvement programme, we decreased LOS, readmissions and postsurgical complications, and improved disposition after discharge in TKA and THA patients. The success of the programme was founded on encompassing the entire process of care, including recognition, readiness, restoration and recovery, on standardising care around evidence-based best practices and Lean efficiencies, and on engaging patients and care givers through shared decision making and other tools.

Contributors LS was responsible for project conceptualisation and day-today implementation of the work, initial manuscript drafting, and final editing and approval of the manuscript. LI was responsible for research design, initial manuscript drafting, and final editing and approval of the manuscript. JS was responsible for conceptualisation and day-to-day implementation of the work, initial manuscript drafting, and final editing and approval of the manuscript. BW was responsible for research design, data analysis, and final editing and approval of the manuscript. RM was responsible for project conceptualisation and final editing and approval of the manuscript. CB was responsible for research design, data analysis, initial manuscript drafting, and final editing and approval of the manuscript.

Funding The authors have not declared a specific grant for this research from any funding agency in the public, commercial or not-for-profit sectors.

Competing interests None declared.

Patient consent for publication Not required.

Provenance and peer review Not commissioned; externally peer reviewed.

Open access This is an open access article distributed in accordance with the Creative Commons Attribution Non Commercial (CC BY-NC 4.0) license, which permits others to distribute, remix, adapt, build upon this work non-commercially, and license their derivative works on different terms, provided the original work is properly cited, appropriate credit is given, any changes made indicated, and the use is non-commercial. See: http://creativecommons.org/licenses/by-nc/4.0/.

\section{REFERENCES}

1. Kenney C. Transforming health care: Virginia Mason Medical Center's pursuit of the perfect patient experience. Boca Raton: CRC Press, 2010.

2. Kaplan GS, Patterson $\mathrm{SH}$, Ching JM, et al. Why Lean doesn't work for everyone. BMJ Qual Saf 2014;23:970-3.
3. Iorio R, Robb WJ, Healy WL, et al. Orthopaedic surgeon workforce and volume assessment for total hip and knee replacement in the United States: preparing for an epidemic. J Bone Joint Surg Am 2008;90:1598-605.

4. Orszag PR, Emanuel EJ, Refrom HC. Health Care Reform and Cost Control. N Engl J Med Overseas Ed 2010;363:601-3.

5. Kurtz S, Ong K, Lau E, et al. Projections of primary and revision hip and knee arthroplasty in the United States from 2005 to 2030. J Bone Joint Surg Am 2007;89:780-5.

6. Lansky D, Milstein A. Quality measurement in orthopaedics: the purchasers' view. Clin Orthop Relat Res 2009;467:2548-55.

7. Ghomrawi HMK, Schackman BR, Mushlin Al. Appropriateness criteria and elective procedures--total joint arthroplasty. N Engl $J$ Med 2012;367:2467-9.

8. Tomek IM, Sabel AL, Froimson MI, et al. A collaborative of leading health systems finds wide variations in total knee replacement delivery and takes steps to improve value. Health Aff 2012;31:1329-38.

9. Bozic KJ, Ward L, Vail TP, et al. Bundled payments in total joint arthroplasty: targeting opportunities for quality improvement and cost reduction. Clin Orthop Relat Res 2014;472:188-93.

10. Levesque J. Satisfaction guaranteed. Seattle Business 2014;72.

11. Ayers DC, Bozic KJ. The importance of outcome measurement in orthopaedics. Clin Orthop Relat Res 2013;471:3409-11.

12. Bozic KJ. Value-based healthcare and orthopaedic surgery. Clin Orthop Relat Res 2012;470:1004-5.

13. Youm J, Chenok K, Belkora J, et al. The emerging case for shared decision making in orthopaedics. J Bone Joint Surg Am 2012;94:1907-12.

14. Russ LR, Phillips J, Brzozowicz K, et al. Experience-based design for integrating the patient care experience into healthcare improvement: Identifying a set of reliable emotion words. Healthc 2013;1:91-9.

15. Bate P, Robert G. Experience-based design: from redesigning the system around the patient to co-designing services with the patient. Qual Saf Health Care 2006;15:307-10.

16. Dr. Robert Bree Collaborative, 2013. Available: http://www. breecollaborative.org/wp-content/uploads/tkrthr_bundle.pdf. [Accessed 13 May 2015].

17. Hanson NA, Allen CJ, Hostetter LS, et al. Continuous ultrasoundguided adductor canal block for total knee arthroplasty: a randomized, double-blind trial. Anesth Analg 2014;118:1370-7.

18. Sukeik M, Alshryda S, Haddad FS, et al. Systematic review and meta-analysis of the use of tranexamic acid in total hip replacement. $J$ Bone Joint Surg Br 2011;93-B:39-46.

19. Pugely AJ, Martin CT, Gao Y, et al. Comorbidities in patients undergoing total knee arthroplasty: do they influence hospital costs and length of stay? Clin Orthop Relat Res 2014;472:3943-50.

20. Gage MJ, Schwarzkopf R, Abrouk M, et al. Impact of metabolic syndrome on perioperative complication rates after total joint arthroplasty surgery. J Arthroplasty 2014;29:1842-5.

21. Zmistowski B, Dizdarevic I, Jacovides CL, et al. Patients with uncontrolled components of metabolic syndrome have increased risk of complications following total joint arthroplasty. J Arthroplasty 2013;28:904-7. 
22. Institute for Healthcare Improvement. Integrated care pathway for total joint arthroplasty. Charlotte, NC and Cambridge, MA; 2013.

23. Arterburn $\mathrm{D}$, Wellman $\mathrm{R}$, Westbrook $\mathrm{E}$, et al. Introducing decision aids at Group Health was linked to sharply lower hip and knee surgery rates and costs. Health Aff 2012;31:2094-104.

24. Ho DM, Huo MH. Are critical pathways and implant standardization programs effective in reducing costs in total knee replacement operations? J Am Coll Surg 2007;205:97-100.

25. Kim S, Losina E, Solomon DH, et al. Effectiveness of clinical pathways for total knee and total hip arthroplasty: literature review. $J$ Arthroplasty 2003;18:69-74.
26. Marshall DA, Christiansen T, Smith C, et al. Continuous quality improvement program for hip and knee replacement. Am J Med Qual 2015;30:425-31.

27. Walter F, Bass N, Bock G, et al. Success of clinical pathways for total joing arthroplasty in a community hospital. Clin Orthop Relate Res 2007;457:133-7.

28. Vorhies JS, Wang Y, Herndon JH, et al. Decreased length of stay after TKA is not associated with increased readmission rates in a national Medicare sample. Clin Orthop Relat Res 2012;470:166-71.

29. Bini SA, Fithian DC, Paxton LW, et al. Does discharge disposition after primary total joint arthroplasty affect readmission rates? $J$ Arthroplasty 2010;25:114-7. 